\title{
Utility of thoracic computed tomography to predict need for early surgery and recurrence after first episode of primary spontaneous pneumothorax
}

\author{
Authors: Asif Azam,, Ahsan Zahid, ${ }^{\mathrm{A}}$ Qaiser Abdullah, ${ }^{\mathrm{A}}$ Noman Qayyum, ${ }^{\mathrm{B}}$ Mostafa Abdelmoteleb ${ }^{\mathrm{B}}$ and \\ Muhammad Badar Ganaie ${ }^{C}$
}

\section{Introduction}

CT imaging is not advocated by British Thoracic Society guidelines after first episode of primary spontaneous pneumothorax (PSP). There is emerging evidence that emphysema-like changes and CT-based dystrophy severity score (DSS) can predict need for early surgery and recurrence.

\section{Objectives}

We aimed to assess the role of DSS during first episodes of PSP in predicting the need for early surgery and recurrence.

\section{Methods}

We conducted a retrospective analysis of consecutive PSP episodes $(n=197)$ admitted to our institution from 1 January 2012 to 31 December 2017. DSS was calculated based on type, number and distribution of blebs and bullae. Patients were categorised as low-grade (0-3) or high-grade (4-6) DSS assessed by a thoracic radiologist.

Results

Forty-five PSP patients had CT at first presentation. Eight patients had low-grade DSS; all were managed non-surgically and none had recurrence over 12 months. Thirty-seven patients had high-grade DSS. Of these, 25 (67.5\%) were managed surgically, with three having contralateral recurrence over 12 months; 12 (32.5\%) were managed non-surgically, and of these two patients had ipsilateral recurrence over 12 months.

Conclusion

DSS seems to predict the need for early surgery and recurrence and CT can be used to risk-stratify patients after a first episode of PSP.

KEYWORDS: Pneumothorax, emphysema, recurrence, radiology, thoracic surgical procedures

DOI: 10.7861/clinmed.2021-0074

\footnotetext{
Authors: Aspecialist registrar in respiratory medicine, Royal Stoke University Hospital, University Hospitals of North Midlands, Stokeon-Trent, UK; ${ }^{\mathrm{B}}$ Consultant radiologist, Royal Stoke University Hospital, University Hospitals of North Midlands, Stoke-on-Trent, UK; ' Consultant respiratory physician, Royal Stoke University Hospital, University Hospitals of North Midlands, Stoke-on-Trent, UK
}

\section{Introduction}

Primary spontaneous pneumothorax (PSP) is the spontaneous buildup of air in the pleural cavity without any trauma. PSP can occur in healthy subjects with no known lung disease, although most have emphysema-like changes (ELCS) predisposing to pneumothorax. ${ }^{1,2}$

The incidence of PSP is 12.3 per 100,000 in males and 2.2 per 100,000 in females, quoted in a recent Danish study. ${ }^{3}$ A UK-based epidemiological study in 2015 quoted 1,804 hospital admissions for PSP in males and 564 in females in the year, leading to an admission-based rate of 8.2 per 100,000 for men and 2.5 per 100,000 for women.

Initial diagnosis of PSP is readily made by conventional chest $X$-ray (CXR). British Thoracic Society (BTS) guidelines advocate CT scanning for uncertain and complex cases only. Needle aspiration (NA) is the treatment of choice for PSP $>2 \mathrm{~cm}$ with significant breathlessness, followed by intercostal chest drain if NA fails. Surgical intervention is considered largely for patients with a second episode of PSP or persistent air leak after 5-7 days of drainage, and for patients with occupations that increase their risk (eg pilots, deep sea divers). ${ }^{5}$ Surgical intervention with or without talc pleurodesis confers the least recurrence risk, between $0-3.2 \%{ }^{6}$

Recurrence of PSP is expected to be around $32 \%$, with the highest risk in the first year after the index event. Risk factors linked with recurrence include smoking, female sex, low body mass index (BMI) and dystrophic lesions on imaging. ${ }^{7}$ Prevention of further recurrent events is an important step in the overall management of PSP. Since PSP is thought to occur due to the rupture of blebs or bullae, the presence of dystrophic lesions on $\mathrm{CT}$ imaging has been a subject of interest as a predictor of recurrence..$^{8-11}$ There have been many attempts to introduce radiological scoring to help with clinical decision making in this regard. The dystrophic severity score (DSS), which was originally proposed by Ouanes-Besbes et al and modified by Casali et al, looks at the number and distribution of blebs and bullae on CT scan. ${ }^{9,10}$

Our study assesses the usefulness of the CT scan and DSS in patients with first presentation of PSP to predict the need for early surgical intervention and recurrence prevention.

\section{Methods}

This was a retrospective observational study of patients presenting to University Hospital of North Midlands (UHNM), Stoke-on-Trent, 
Table 1. Dystrophy severity score (adapted from Primavesi et al). ${ }^{11}$

$\begin{array}{llll}\begin{array}{l}\text { CT-based } \\ \text { dystrophic } \\ \text { lesions }\end{array} & \text { 0 point } & \mathbf{1} \text { point } & \mathbf{2} \text { points } \\ \begin{array}{l}\text { Type } \\ \text { Number }\end{array} & \text { No lesion } & \text { Blebs }(<1 \mathrm{~cm}) & \text { Bullae }(>1 \mathrm{~cm}) \\ \text { Laterality } & \text { No lesion } & \text { Single } & \text { Multiple } \\ \text { DSS grades } & \text { Unilateral } & \text { Bilateral } \\ \text { Low grade } & & & \\ \text { High grade } & & 0-3 & \\ \end{array}$

between January 2012 and December 2017 with first episode of PSP ( $n=197)$. Patients were identified using the International Classification of Diseases, 10th revision (ICD-10). Patients with underlying lung disease or age above 50 years (secondary spontaneous pneumothorax), traumatic pneumothorax, post-operative pneumothorax and iatrogenic pneumothorax were excluded.

PSP patients who had CT scans during the admission were included for further analysis. CT images on picture archiving and communication system (PACS) were reviewed by an independent thoracic radiologist who was blinded to eventual patient outcomes. DSS was calculated from CT scan based on the type, number and distribution of blebs and bullae and patients were grouped as lowgrade DSS (score 0-3, Fig 1) or high-grade DSS (score 4-6; Fig 2, Table 1). ${ }^{11}$

Outcome measures, obtained from patient care records, included length of stay (LOS), surgical intervention and recurrence rate within 1 year. Categorical variables ( $n$, \%) were evaluated using Fisher's exact test. Continuous data (median, interquartile range) were compared using the Wilcoxon rank sum test. P-values $<0.05$ were considered statistically significant.

Ethical approval and informed consent were not obtained as no patient information or patient identifiable data or figures were included in this article.

\section{Results}

Forty-five PSP patients (23\%) had CT scans during the first presentation. Indications for CT scan included persistent air leak $(n=26)$ and identification of underlying cause of pneumothorax including bullae $(n=19)$. The median age of the patients was 31 years; $82 \%$ were male and $73 \%$ of patients were smokers.

Eight patients were classified as low-grade and 37 as highgrade based on the basis of DSS score. There were no statistically significant differences between groups in terms of age, sex and smoking history (Table 2).

All patients with low grade DSS ( $n=8)$ were managed non-surgically and none had recurrence over 12 months.

Twenty-five of the 37 high grade DSS patients (67.5\%) underwent surgical intervention (18 as inpatients). Three of these patients had contralateral recurrence of pneumothorax over the ensuing 12 months. The other 12 high-grade patients (32.5\%) were managed conservatively, and two patients had ipsilateral recurrence over the next 12 months.

Patients in the high-grade DSS group had significantly high rates of surgical intervention when compared to the low grade DSS group (67.5\% versus $0 \%, p=0.0006)$. There was a trend towards higher

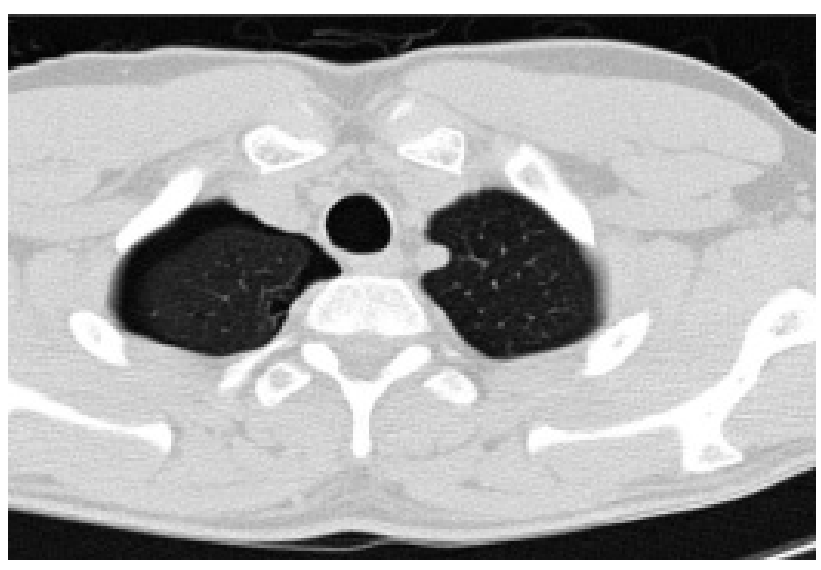

Fig 1. CT scan of patient with low-grade dystrophy severity score (DDS).

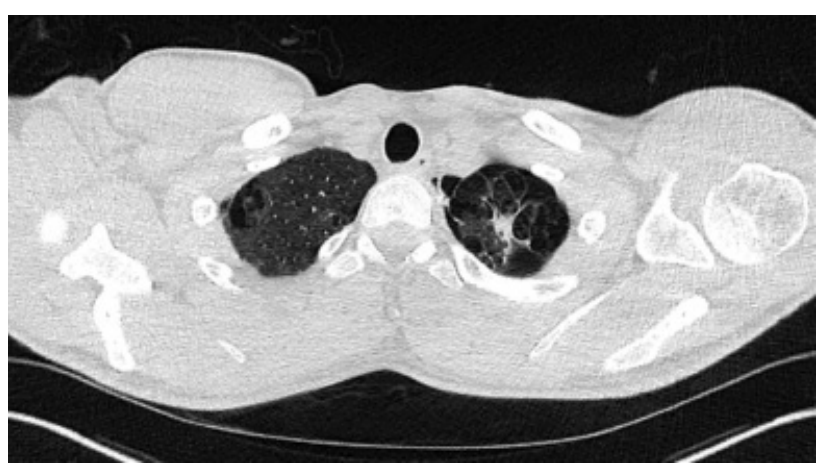

Fig 2. CT scan of patient with high-grade dystrophy severity score (DDS).

LOS ( 8 days versus 5 days, $p=0.52$ ) and increased recurrence rates at 12 months $(13.5 \%$ versus $0 \%, p=0.57)$ in the high grade DSS group, although there was no statistical significance.

\section{Discussion}

Our study looked at the usefulness of $\mathrm{CT}$ imaging to anticipate the need for early surgery and recurrence after initial presentation with PSP. We showed that the presence of dystrophic lesions on CT predicts the need for early surgery and possibly recurrence within the subsequent 12 months. CT scan after the initial episode of PSP can detect the severity of underlying ELCs, identify patients at risk of failure of conservative treatment and potentially prevent recurrence by indicating the need to carry out early ipsilateral surgical intervention. Patients can be offered staged contralateral surgery or surveillance in the pleural clinic. We propose an algorithm for the management of first episode of PSP (Fig 3).

PSP by definition has no underlying cause, but studies have shown rupture of dystrophic lesions or air leak from pleural porosities as the likely contributing factors. ${ }^{12,13}$ Early surgical intervention can prevent further recurrence, especially for high-risk patients identified through CT imaging. ${ }^{14}$

Previous studies have suggested that the number and size of blebs and bullae on $\mathrm{CT}$ imaging are directly proportional to the recurrence of PSP and eventual need for surgical intervention. ${ }^{8,10,11}$ A recent multicentre randomised controlled trial by Olesen et al compared the role of conventional intercostal drain with surgical management after the first episode of PSP. They concluded that surgery was 
Table 2. Comparison of low-grade DSS with high-grade DSS in predicting early surgical intervention and rate of recurrence after first episode of primary spontaneous pneumothorax (PSP).

Low-grade DSS $(n=8)$

Median age, years (IQR)

Male, n (\%)

Right sided, $\mathbf{n}(\%)$

Current/ex-smoker, n (\%)

Median LOS, days (IQR)

Surgical intervention, $\mathrm{n}(\%)$

Recurrence at 1 year, $\mathrm{n}(\%)$
$35(23.5-46.7)$

$8(100 \%)$

$3(37.5 \%)$

$5(62.5 \%)$

$5(3.2-8.5)$

0

0
High-grade DSS ( $n=37)$

$31(24-34.5)$

$29(78 \%)$

$17(46 \%)$

$28(75.7 \%)$

$8(4-12)$

$25(67.5 \%)$

$5(13.5 \%)$

Palue

0.51

0.32

0.72

0.66

0.52

0.0006

0.57

DSS = dystrophy severity score; IQR = interquartile range; $L O S$ = length of stay.

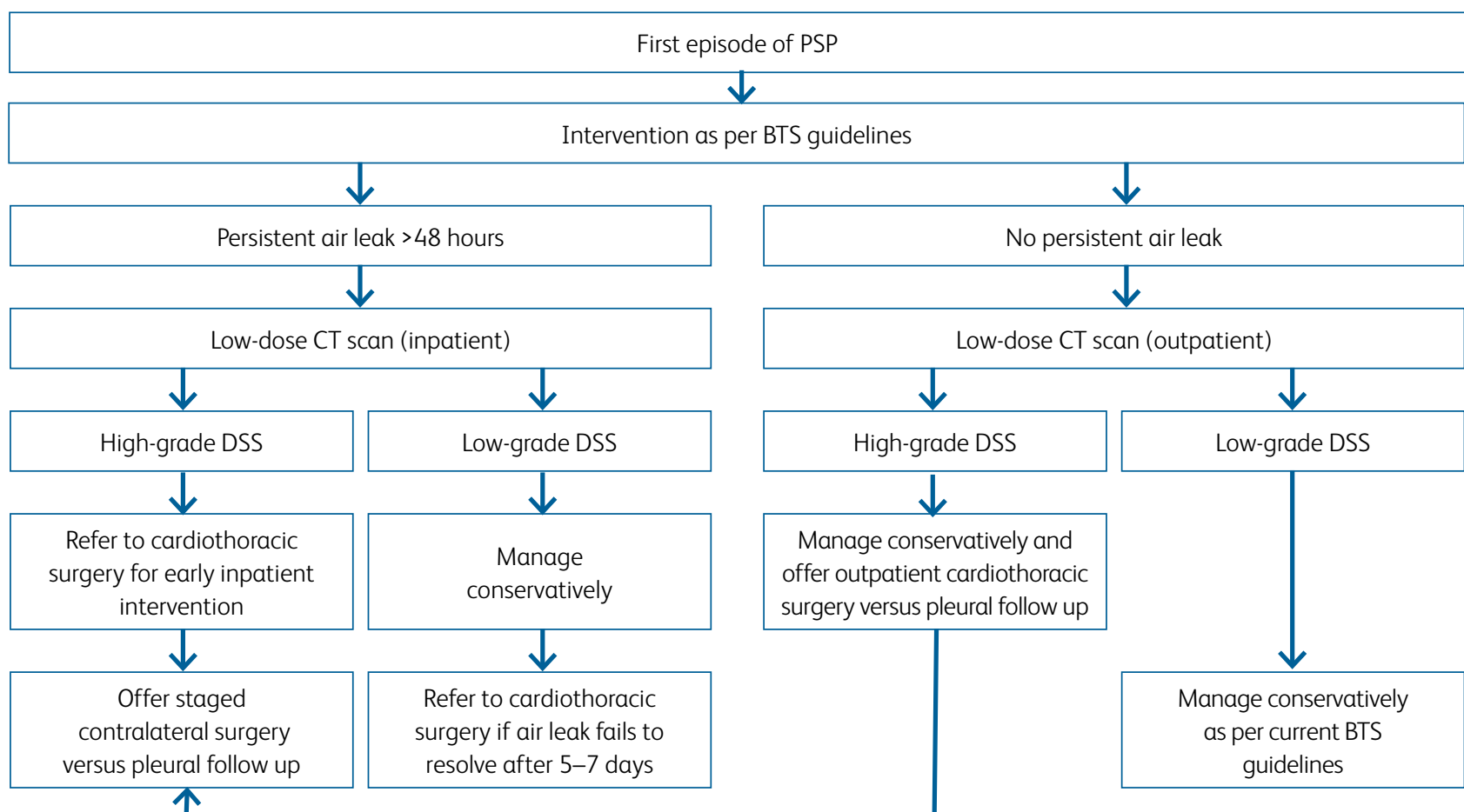

Fig 3. Proposed algorithm for management of first episode of primary spontaneous pneumothorax (PSP). BTS = British Thoracic Society; DSS = dystrophy severity score

effective in preventing recurrence after the first episode of PSP and should be the standard of care when CT shows high-grade dystrophic lesions, specifically bullae $>2 \mathrm{~cm}$. ${ }^{15}$ Previous studies have shown that the time to recurrence is significantly shorter in patients managed conservatively as compared to those managed with surgical intervention. ${ }^{11}$

Existing international guidelines do not support surgical intervention to prevent recurrence after first episode of PSP. 5,16,17 It is undetermined whether surgery should be considered after first episode of PSP especially in those at high risk of recurrent episodes, including females, patients with low BMI and those with dystrophic lesions on $\mathrm{CT} .{ }^{18}$ Recently published large multicentre randomised controlled trials have revealed the usefulness of a conservative approach and ambulatory drainage for management of PSP. ${ }^{19,20}$
BTS guidelines are due an update shortly and will likely address age criteria for PSP and optimal management after first episode of PSP, including simple observation versus intervention and the role of imaging and surgery.

Up to $10 \%$ of patients presenting with PSP have causative diffuse cystic lung disease (DCLD), such as Birt-Hogg-Dubé syndrome, lymphangioleiomyomatosis or pulmonary Langerhans cell histiocytosis. High-resolution CT (HRCT) screening of PSP patients for DCLDs has been shown to be cost-effective. ${ }^{21}$ Likewise, around $10 \%$ of PSP patients have a family history of pneumothorax that could point towards an underlying genetic disorder. ${ }^{22}$ Some authors suggest CT imaging after first episode of PSP in female patients and those with family history of pneumothorax. ${ }^{23}$ 


\section{Limitations}

Our study has a few limitations that must be borne in mind.

This study was a single-centre retrospective observational study, with all the known drawbacks of retrospective data collection. Patient data were compiled from case notes and electronic patient records and the results could have been influenced by the exclusion of unmeasured confounding variables.

A major limitation of our data is the small study size, with most CT scans conducted in high-grade DSS group for patients with non-resolving air leak, hence leading to uneven distribution between groups and potentially selection bias. Further prospective randomised controlled trial is needed in a larger cohort to corroborate these results.

\section{Conclusion}

CT based DSS seems to predict the need for early surgery and recurrence after first episode of PSP. CT can be used to risk stratify patients after first episode of PSP and identify patients at high risk of failure of conventional treatment and early recurrence. CT imaging can also potentially uncover an underlying diffuse cystic lung disease or a genetic disorder. Further prospective randomised studies are required to validate these findings.

\section{References}

1 Lesur O, Delorme N, Fromaget JM, Bernadac P, Polu JM. Computed tomography in the etiologic assessment of idiopathic spontaneous pneumothorax. Chest 1990;98:341-7.

2 Bintcliffe OJ, Edey AJ, Armstrong L, Negus IS, Maskell NA. Lung parenchymal assessment in primary and secondary pneumothorax. Ann Am Thorac Soc 2016;13:350-5.

3 Olesen WH, Titlestad IL, Andersen PE, Lindahl-Jacobsen R, Licht PB. Incidence of primary spontaneous pneumothorax: a validated, register-based nationwide study. ERJ Open Res 2019;5:00022-2019.

4 Hallifax RJ, Goldacre R, Landray MJ, Rahman NM, Goldacre MJ. Trends in the incidence and recurrence of inpatient-treated spontaneous pneumothorax, 1968-2016. JAMA 2018;320:1471-80.

5 MacDuff A, Arnold A, Harvey ] et al. Management of spontaneous pneumothorax: British Thoracic Society Pleural Disease Guideline 2010. Thorax 2010;65 Suppl 2:ii18-31.

6 Hallifax RJ, Yousuf A, Jones HE et al. Effectiveness of chemical pleurodesis in spontaneous pneumothorax recurrence prevention: a systematic review. Thorax 2017;72:1121-31.

7 Walker SP, Bibby AC, Halford P et al. Recurrence rates in primary spontaneous pneumothorax: a systematic review and metaanalysis. Eur Respir J 2018;52:1800864.

8 Warner BW, Bailey WW, Shipley RT. Value of computed tomography of the lung in the management of primary spontaneous pneumothorax. Am J Surg 1991;162:39-42.
9 Ouanes-Besbes L, Golli M, Knani ] et al. Prediction of recurrent spontaneous pneumothorax: $C T$ scan findings versus management features. Respir Med 2007;101:230-6.

10 Casali C, Stefani A, Ligabue G et al. Role of blebs and bullae detected by high-resolution computed tomography and recurrent spontaneous pneumothorax. Ann Thorac Surg 2013;95:249-55.

11 Primavesi F, Jäger T, Meissnitzer T et al. First episode of spontaneous pneumothorax: CT-based scoring to select patients for early surgery. World J Surg 2016;40:1112-20.

12 Noppen M, Dekeukeleire T, Hanon S et al. Fluorescein-enhanced autofluorescence thoracoscopy in patients with primary spontaneous pneumothorax and normal subjects. Am J Respir Crit Care Med 2006;174:26-30.

13 Muramatsu T, Shimamura M, Furuichi M et al. Cause and management of recurrent primary spontaneous pneumothorax after thoracoscopic stapler blebectomy. Asian J Surg 2011;34:69-73.

14 Chen YY, Huang HK, Chang H, Lee SC, Huang TW. Postoperative predictors of ipsilateral and contralateral recurrence in patients with primary spontaneous pneumothorax. J Thorac Dis 2016;8:3217-24.

15 Olesen WH, Katballe N, Sindby JE et al. Surgical treatment versus conventional chest tube drainage in primary spontaneous pneumothorax: a randomized controlled trial. Eur J Cardiothorac Surg 2018;54:113-21.

16 Baumann MH, Strange C, Heffner JE et al. Management of spontaneous pneumothorax: an American College of Chest Physicians Delphi consensus statement. Chest 2001;119:590-602.

17 Tschopp JM, Bintcliffe O, Astoul P et al. ERS task force statement: diagnosis and treatment of primary spontaneous pneumothorax. Eur Respir ] 2015;46:321-35.

18 Porcel JM. Phenotyping primary spontaneous pneumothorax. Eur Respir ] 2018;52: 1801455.

19 Brown SGA, Ball EL, Perrin K et al. Conservative versus interventional treatment for spontaneous pneumothorax. N Engl J Med 2020;382:405-15.

20 Hallifax RJ, McKeown E, Sivakumar P et al. Ambulatory management of primary spontaneous pneumothorax: an open-label, randomised controlled trial. Lancet 2020;396:39-49.

21 Gupta N, Langenderfer D, McCormack FX, Schauer DP, Eckman $\mathrm{MH}$. Chest computed tomographic image screening for cystic lung diseases in patients with spontaneous pneumothorax is cost effective. Ann Am Thorac Soc 2017;14:17-25.

22 Scott RM, Henske EP, Raby B et al. Familial pneumothorax: towards precision medicine. Thorax 2018;73:270-6.

23 Boone PM, Scott RM, Marciniak SJ, Henske EP, Raby BA. The genetics of pneumothorax. Am J Respir Crit Care Med 2019;199:1344-57.

Address for correspondence: Dr Muhammad Badar Ganaie, Department of Respiratory Medicine, Royal Stoke University Hospital, University Hospitals of North Midlands, Newcastle Road, Stoke-on-Trent ST4 6QG

Email:muhammad.ganaie@uhnm.nhs.uk

Twitter: @badar_ganaie 